\title{
Relação entre os pensamentos ruminativos e índices de ansiedade e depressão em estudantes de psicologia
}

\section{Comparison between ruminative thoughts and indexes of anxiety and depression in psychology students}

\author{
Marilia Lopes Oliveira Bezerra', Gustavo Marcelino Siquara'², José Neander Silva Abreu ${ }^{3}$ \\ ${ }^{1}$ Autora para correspondência. Escola Bahiana de Medicina e Saúde Pública. Salvador, Bahia, Brasil. ORCID: 0000-0003-3155-8990. \\ marilialopesbezerra@gmail.com \\ 2Escola Bahiana de Medicina e Saúde Pública. Salvador, Bahia, Brasil. ORCID: 0000-0002-4495-6835. gustavosiquara@bahiana.edu.br \\ ${ }^{3}$ Universidade Federal da Bahia. Salvador, Bahia, Brasil. ORCID: 0000-0001-7636-3666. neandersa@hotmail.com
}

\begin{abstract}
RESUMO I O período universitário é marcado por descobertas e desafios que podem servir como gatilho para o desenvolvimento de transtornos de humor e ansiedade. A sucessão de fatores estressores é observada em estudantes de diversas nacionalidades e culturas, destacando-se o papel dos pensamentos ruminativos e sua prevalência em indivíduos propensos a estados de humor deprimido. $O$ presente estudo realiza uma análise transversal em 166 estudantes de psicologia de 03 instituições de ensino superior da Cidade de Salvador, na Bahia, através da aplicação de escalas psicométricas sendo elas: Questionário de Ruminação e Reflexão (QRR), Inventário de Ansiedade Traço-Estado (IDATE) e Inventário Beck de Depressão (BDI). Os dados obtidos foram analisados através do programa estatístico SPSS 20.0. Para as análises foram utilizadas ferramentas de estatísticas descritiva e inferencial como a correlação de Sperman e teste $t$ de student. A ansiedade e depressão apresentam forte correlação, com a escala de pensamentos ruminativos. Os níveis clínicos de ansiedade e depressão estiveram acima do que a literatura aponta ao comparar com a população geral. $O$ acometimento dos estudantes universitários por ansiedade e depressão gera grande impacto na produtividade e inserção profissional, e os prejuízos relacionados a estes transtornos extrapolam para o meio social onde os mesmos estão inseridos. Por isso, torna-se necessária a adoção de medidas preventivas, que permitam um maior equilíbrio emocional e psíquico desses indivíduos.
\end{abstract}

PALAVRAS-CHAVE: depressão, ansiedade, pensamentos ruminativos, estudantes universitários, Psicologia.

\begin{abstract}
I The university period is marked by discoveries and challenges that can serve as triggers for the development of mood and anxiety disorders. The succession of stressors is observed in students of diverse nationalities and cultures, highlighting the role of ruminal thoughts and their prevalence in individuals prone to depressed mood states. A cross-sectional analysis was carried out in 166 students from 03 higher education institutions in the City of Salvador, Bahia, through the use of psychometric scales such as Questionnaire of Rumination and Reflection (QRR), TraitState Anxiety Inventory ) And Beck Depression Inventory (BDI), obtaining quantitative data, analyzed statistically through SPSS 20.0 by the Pearson model. The anxiety and depression present high prevalence in the studied sample, reinforcing the hypothesis widely ratified by the scientific literature, its strong association with rumination and the presence of stressors in the academic scope. The involvement of university students due to anxiety and depression generates great impact on productivity and professional insertion, and the damages related to these disorders extrapolate to the social environment where they are inserted. Therefore, it is necessary to adopt preventive measures that allow a greater emotional and psychic balance of these individuals.
\end{abstract}

KEY WORDS: depression, anxiety, ruminative thoughts, university students, Psychology. 


\section{Introdução}

O período acadêmico durante a Universidade é marcado por descobertas e desafios que precisam ser superados diariamente. Adquirir novos conhecimentos, desenvolver raciocínio crítico em relação ao meio inserido e conviver em grupo, podem ser ações recompensadoras, desafiadoras ou até mesmo gerar adoecimento. A forma como os estudantes enfrentam os obstáculos da vida acadêmica e a própria exposição a situações novas, apresentam um potencial estressor, que podem servir como gatilho para o desenvolvimento de problemas de saúde mental.

Estudantes universitários de nacionalidades e culturas diversas apresentam risco para desenvolver transtornos de saúde mental, devido à sucessão de possiveis estressores. O conceito de saúde mental utilizado, está ligado a resiliência emocional que nos permite desfrutar dos momentos vividos, sobreviver à dor, ao desapontamento, tristeza, permitindo a realização dos papeis que desempenhamos na sociedade. (Khaldoun, Aldiabat, Nasir \& Matani, et al, 2014).

A Organização Mundial de Saúde (OMS) sugere que prejuízos na saúde mental estão associados à fatores de risco como: "mudanças sociais rápidas, condições de trabalho estressantes, discriminação de gênero, exclusão social, estilo de vida pouco saudável, riscos de violência, mal-estar físico e violações dos direitos humanos" (Gallagher, 2008). A maioria desses fatores de risco estão presentes no ambiente universitário.

Condições de estudo (trabalho), como: falta de professores; condições estruturais desfavoráveis; gestores impositivos e pouco assertivos; cobrança das atividades em curtos períodos; situações de discriminação racial; gênero; ausência de hábitos alimentares saudáveis e pratica de exercício físico, comumente é derivado da intensa rotina de estudos; atividade empregatícia associada; conflitos entre os colegas; são alguns dos fatores encontrados ao frequentar esse novo ambiente. $O$ ingresso na vida universitária e os potenciais estressores podem ser entendidos como preditores ambientais para o aumento dos índices de traços para a ansiedade $e$ depressão interferindo na qualidade de vida, saúde física, humor, sono, alimentação, planejamento e produtividade.

Guthrie, Black, Shaw, Hamilton, Creed \& Tomenson (1995), apontam que a forma que o processo educacional é desenvolvido na vida Universitária pode ter um efeito negativo na saúde mental dos alunos, com o aumento da taxa de traços depressivos, ansiosos e sintomas de estresse entre os estudantes de medicina e em outros cursos.

O período de transição entre o ensino médio e a graduação é um momento crítico, no qual o jovem precisa se tornar mais ativo no seu processo de formação universitária, aumentando o nível de exigência, autonomia e criticidade. Nesse momento o estudante torna-se responsável por suas escolhas e decisões, o que frequentemente gera insegurança, dúvidas, medo e abre portas para diversos fatores que podem interferir negativamente em suas trajetórias. Trata-se de um período de transição da adolescência para vida adulta, sendo necessário ingressar no curso superior, manter boas notas, planejar o futuro e muitas vezes ficar longe de casa, podendo acarretar em sintomas de ansiedade (preocupação excessiva) e estresse (quebra da homeostase corporal e psíquica).

Buchanan, (2012) sugere que a escolha pelo curso comumente vem acompanhada de angústias, dúvidas e cobranças sociais, uma vez que se associa a um novo estilo de vida, investimento de tempo e dinheiro que impactarão na vida profissional e pessoal futura. $O$ mundo universitário requer dos estudantes um alto grau de maturidade e responsabilidade podendo não ser correspondidos devido a pensamentos e sentimentos de incapacidade, incertezas e cobranças, ocasionando tristeza e mal-estar. Segundo Storrie et al (2010), "dada a prevalência global e o ônus da doença mental, é provável que haja um número significativo de estudantes com problemas emocionais matriculados na universidade com diagnóstico formal e também sem diagnóstico, mas com sintomas incapacitantes". 
As características mais típicas do estado depressivo são proeminência dos sentimentos de tristeza ou vazio e perda da capacidade de experimentar prazer nas atividades. Alterações cognitivas, redução do interesse pelo ambiente, cansaço exagerado, fadiga, apatia e ansiedade podem derivar deste estado inicial, agravando as consequências e comprometendo a qualidade de vida do sujeito (Del Porto, 1999).

A ruminação é uma forma amplamente estudada de cognição perseverante que é considerada como importante fator etiológico e de manutenção para depressão, enquanto a maleabilidade emocional permite que os estados afetivos transitem de acordo com as experiências vivenciadas (Kuppens, Allen, \& Sheeber, 2010).

Segundo Thomsen (2006) e Kuppens, Allen, \& Sheeber (2010), a ruminação se relaciona mais intensamente com a tristeza quando comparada a outros tipos de afeto negativo. As pessoas que ruminam habitualmente em resposta a um sofrimento, tendem a exibir dependência em suas experiências afetivas negativas e em seus comportamentos. A ruminação pode ser um quadro mal adaptativo em que o indivíduo desenvolve estratégias ineficazes para regular o efeito negativo gerado por determinadas situações. Os pensamentos negativos impactam no rendimento dos estudantes uma vez que podem comprometer a sua saúde mental, elevando níveis de ansiedade, depressão e diminuindo a sua reflexão em relação às atividades da vida social e acadêmica. Assim sendo, é necessário a criação de ambientes acadêmicos amigáveis e afetivos a fim de reduzir estes problemas e suas repercussões na vida dos indivíduos (Campbell-Sills \& Barlow, 2007, Nolen-Hoeksema et al., 2008).

MacKean (2011) e Gallagher (2008) sugerem em seus estudos que os problemas de saúde mental em estudantes universitários como depressão, ansiedade, pensamentos suicidas, psicose, vícios, uso de medicamentos psiquiátricos e outros distúrbios crônicos, estão mais prevalentes quando comparados a população geral. As doenças mentais não diagnosticada ou não tratadas em estudantes universitários podem gerar consequências negativas sobre $\circ$ indivíduo, a família e a comunidade, como alto risco de prejudicar a si próprio ou terceiros. Abandonar os programas educativos desempenhados, aumentar a taxa de desemprego, gerar encargos adicionais para as famílias e sociedade, criando inúmeros problemas psicossociais.

Pode-se desfrutar de bem-estar físico e mental a despeito de um transtorno mental diagnosticado e em processo de tratamento. Para isto, é necessária melhor investigação dos dados epidemiológicos em saúde mental, possibilitando o desenvolvimento de programas de prevenção e tratamento daqueles que possuem os transtornos. Esta pesquisa teve como objetivo avaliar aspectos da saúde mental, ansiedade e depressão, em estudantes Universitários de Psicologia, relacionando com o grau de pensamentos ruminativos.

\section{Método}

\section{Procedimentos}

A composição da amostra foi por conveniência, os estudantes foram convidados pelos pesquisadores do presente estudo a preencher as escalas solicitadas, descritas abaixo. A aplicação das escalas foi feita de forma coletiva após a assinatura do Termo de Consentimento Livre e Esclarecido, com linguagem clara e objetiva.

\section{Participantes}

Participaram da pesquisa 166 estudantes de Psicologia das seguintes instituições de ensino superior: Escola Bahiana de Medicina e Saúde Pública (EBMSP), Universidade Estadual da Bahia (UNEB) e Universidade Federal da Bahia (UFBA), situadas na cidade de Salvador. Os dados descritivos dos participantes podem ser visualizados na Tabela 1. Os critérios para inclusão na amostra foram estudantes matriculados e que frequentam os centros acadêmicos acima descritos. 
Tabela 1. Dados dos participantes da pesquisa

\begin{tabular}{|c|c|c|c|c|}
\hline & & $M(D p)$ & Amplitude & $\mathrm{N}$ \\
\hline & Idade & $21,6(5,5)$ & 17 a 57 & \\
\hline \multirow[t]{2}{*}{ Sexo } & Mulher & & & 126 \\
\hline & Homem & & & 40 \\
\hline \multirow[t]{2}{*}{ Tipo de Instituição } & Pública & & & 94 \\
\hline & Particular & & & 72 \\
\hline \multirow[t]{2}{*}{ Período do Curso } & $\begin{array}{l}\text { Início Curso - } \\
\text { Até } 5^{\circ} \text { semestre }\end{array}$ & & & 88 \\
\hline & $\begin{array}{l}\text { Fim de Curso - } \\
\text { Após } 6^{\circ} \text { semestre }\end{array}$ & & & 78 \\
\hline Total Participantes & & & & 166 \\
\hline
\end{tabular}

Dentre os estudantes que participaram do estudo, 126 são mulheres e 40 são homens, com idade média de 21,6 , sendo a menor idade 17 anos e a maior 57 anos. O projeto obedeceu às questões éticas, sendo aprovado pelo Comitê de Ética em Pesquisa da UNEB, sob número de CAAE: 47512015.0 .0000 .0057 e parecer final de aprovação sob número 1.333.710.

\section{Instrumentos}

\section{Questionário de Ruminação e Reflexão (QRR)}

Composto por duas escalas de 12 itens cada, foi criado para avaliar o quanto os indivíduos engajam-se em pensamentos ruminativos e reflexivos. Alguns exemplos de itens de ruminação são: "Eu passo um bom tempo lembrando momentos constrangedores ou frustrantes pelos quais passei."; "Muito depois de uma discordância ou discussão ter acabado, meus pensamentos continuam voltados para $\circ$ que aconteceu." e "Eu sempre pareço estar remoendo, em minha mente, coisas recentes que disse ou fiz".

Zanon \& Teixeira, 2006; Zanon \& Hutz, (2009) apontam que $\circ Q R R$ apresenta validade de construto e índices de consistência interna satisfatórios para o uso do instrumento em universitários brasileiros ( $a=0,87$ para ambas as escalas). Na ruminação o indivíduo tem uma tendência a empregar excessiva atenção, de forma automática, à eventos ou sentimentos negativos, já na reflexão ele pensa sobre si mesmo, buscando autoconhecimento, podendo ser positivo ou prejudicial para o self. As questões: $6,9,10,13,14,17,20,24$, foram invertidas na escala Likert.

\section{Inventário de Ansiedade Traço-Estado (IDATE)}

É um instrumento de autoavaliação para mensuração da ansiedade e seus subtipos, traço e estado, com questões de múltipla escolha. É composta de 20 questões em cada escala de ansiedade (traço e estado), com escores variando de 1 a 4, e escore total variando de 20 a 80 em cada escala. No momento de aplicação do questionário, deve-se explicar ao sujeito sobre a divisão em (I - IDATE Estado e II IDATE Traço) e em que cada uma consiste. $O$ estado de ansiedade seria uma condição emocional transitória, variando sua intensidade com o perigo percebido pelo indivíduo. Já no traço de ansiedade haveria uma intensificação do estado de ansiedade. Deve ser dito que as respostas não têm como finalidade ser certas ou erradas, e suas respostas devem estar de acordo com as opções ofertadas.

Com relação à quantificação e interpretação das respostas, atribui-se pontuação previamente definidas para cada quesito. Os escores para as perguntas de caráter positivo são invertidos, ou seja, se $\circ$ paciente responder 4 , atribui-se $\circ$ valor $1 \mathrm{na}$ codificação; se responder 3, atribui-se o valor 2; se responder 2, atribui-se o valor 3 , se responder 1 , atribui-se $\circ$ valor 4 . Para $\circ$ IDATE-estado, as per- 
guntas negativas são: $3,4,6,7,9,12,13,14,17$, 18; e as positivas: $1,2,5,8,10,11,15,16,19,20$. Para o IDATE- traço, as perguntas negativas são: 2 , $3,5,8,9,11,12,15,15,17,18,20$; e as positivas: $1,6,7,10,13,16,19$. Foi utilizada a análise Rasch para a simplificação do instrumento original e para torná-lo transcultural, independente de gêneros e circunstâncias, permitindo o uso de intervenções para redução de ansiedade.

\section{Inventário Beck de Depressão:}

Inventário de Depressão de Beck (BDI): é um instrumento de autorrelato constituído por 21 grupos de afirmações. Cada um desses grupos possui quatro afirmações em que a pessoa deve responder qual a opção que esta de acordo com o seu estado naquela determinada situação. Estas afirmações estão relacionadas com sinalizações dos sintomas referentes a estados depressivos do indivíduo e possibilidade diagnostica.

\section{Análise de dados}

Os dados foram analisados utilizando-se procedimentos estatísticos de análise descritiva, correlacional e teste de hipóteses. Os dados foram tabulados no software estatístico SPSS 20.0. A análise de correlação foi feita através do modelo de Pearson.

Essas técnicas foram utilizadas para verificar o grau de associação entre pensamentos ruminativos e os índices de ansiedade e sintomas depressivos em estudantes de Psicologia.

\section{Resultados}

Serão apresentados os resultados das escalas de ansiedade, depressão e ruminação nas pessoas avaliadas e as correlações entre as escalas.

Tabela 2. Descrição dos dados que indicam as pontuações encontradas nas escalas.

\begin{tabular}{llllll}
\hline & $\begin{array}{l}\text { BDI } \\
\mathrm{N}=163\end{array}$ & $\begin{array}{l}\text { IDATE } \\
\text { Traço } \\
\mathrm{N}=153\end{array}$ & $\begin{array}{l}\text { IDATE } \\
\text { Estado } \\
\mathrm{N}=164\end{array}$ & Ruminação & Reflexão \\
\hline Média (Dp) & $13,7(9,3)$ & $47,1(11,5)$ & $43.53(9,5)$ & $43,63(8,8)$ & $43,44(7,3)$ \\
Min. - Max. & $0,0-40$ & $25-74$ & $22-71$ & $18-58$ & $19-60$ \\
Mínimo (0-13) & $92(56 \%)$ & & \\
Leve (14-19) & $30(18 \%)$ & & & \\
Moderado (20-28) & $25(15 \%)$ & & & & \\
Grave (acima 29) & $16(9 \%)$ & & & & \\
Leve (até 33) & & $13(8 \%)$ & $30(18 \%)$ & \\
Média (33-49) & & $76(49 \%)$ & $89(54 \%)$ & & \\
Alta (acima 49) & & $64(42 \%)$ & $45(27 \%)$ & \\
\hline
\end{tabular}

BDI - Inventário de Depressão de Beck - IDATE - Inventário de Ansiedade Traço-Estado QRR- Questionário de Ruminação e Reflexão

A análise dos resultados da Escala de Ansiedade Traço - Estado sugerem que $8 \%$ - 18\% da amostra apresentaram nível leve, em cada escala, $49 \%$ e $54 \%$ apresentaram nível médio respectivamente, enquanto $42 \%$ e $27 \%$ apresentaram nível alto em cada escala descritos na tabela 2 .
Os resultados da pesquisa sugerem que $56 \%$ da amostra apresentam níveis mínimo de sintomas de depressão, seguidos de $18 \%$ apresentando níveis leve de sintomas depressivo, 15\% moderado e 9\% da amostra apresentaram nível grave de sintomas de depressão segundo normatização do instrumento utilizado. 
Tabela 3. Correlação Bivariada de Sperman entre as escalas de Depressão, Ansiedade e Ruminação

\begin{tabular}{|c|c|c|c|c|}
\hline & Ruminação & IDATE Estado & IDATE Traço & $\mathrm{BDI}$ \\
\hline Ruminação & - & $0.508 * *$ & $0.692 * *$ & $0.444^{* *}$ \\
\hline $\begin{array}{l}\text { IDATE } \\
\text { Estado }\end{array}$ & & - & $0.660 * *$ & $0.637^{* *}$ \\
\hline IDATE Traço & & & - & $0.771 * *$ \\
\hline BDI & & & & - \\
\hline
\end{tabular}

A Tabela 3 apresenta as correlações totais de Ruminação, IDATE-E, IDATE-T e BDI em estudantes do curso de Psicologia. A partir dos resultados observa-se maior significância estatística na correlação entre a Ruminação e IDATE-T $(r=0,692, p<0,01)$, assim como BDI $(r=0,444, p<0,01)$ e IDATE-E $(r=0,508, p<0,01)$. Destaca-se na tabela a força da associação positiva entre os níveis de ansiedade (IDATE-T) e depressão (BDI) $(r=0,771, p<0,01)$.

Tabela 4. Comparação entre características dos participantes

\begin{tabular}{|c|c|c|c|c|c|c|c|c|}
\hline & BDI & $\begin{array}{c}P \\
\text { valor }\end{array}$ & $\begin{array}{l}\text { IDATE } \\
\text { - Traço }\end{array}$ & $\begin{array}{c}P \\
\text { valor }\end{array}$ & $\begin{array}{l}\text { IDATE } \\
\text { - Estado }\end{array}$ & $\begin{array}{c}P \\
\text { valor }\end{array}$ & Ruminação & $\begin{array}{l}P \\
\text { valor }\end{array}$ \\
\hline $\begin{array}{l}\text { Inicio - } \\
\text { até } 5 \text { sem. }\end{array}$ & $14,2(9,4)$ & 0,801 & $47,3(10,5)$ & 0,918 & $43,9(10,4)$ & 0,808 & $43,5(7,5)$ & 0,382 \\
\hline $\begin{array}{c}\text { Final - } \\
\text { após } 6 \text { sem. }\end{array}$ & $14,5(10,8)$ & & $47,5(12,4)$ & & $43,4(11,4)$ & & $42,3(9,3)$ & \\
\hline Pública & $14,9(9,2)$ & 0,246 & $48,6(10,3)$ & 0,108 & $44,6(10,5)$ & 0,184 & $43,1(7,9)$ & 0,638 \\
\hline Particular & $13,1(11,0)$ & & $45,6(12,6)$ & & $42,3(11,2)$ & & $42,5(9,0)$ & \\
\hline Homem & $13,7(9,1)$ & 0,762 & $45,2(11,8)$ & 0,261 & $43,5(11,7)$ & 0,939 & $42,0(8,5)$ & 0,500 \\
\hline Mulher & $14,3(10,3)$ & & $47,7(11,4)$ & & $43,6(10,6)$ & & $43,1(8,3)$ & \\
\hline
\end{tabular}

Não foram encontradas diferenças significativas ao comparar os sujeitos da amostra quanto ao início e final do curso, instituição pública e particular e gênero.

\section{Discussão}

Os objetivos do estudo foi avaliar os aspectos da saúde mental (ansiedade e depressão) em estudantes Universitários, relacionando com $\circ$ grau de pensamentos ruminativos. A saúde mental de estudantes universitários tem sido foco de estudos cien- tíficos, devido ao aumento dos níveis de ansiedade, depressão, uso de medicamentos psiquiátricos, uso abusivo de substâncias e risco de suicídio nesta população (MacKean, 2011 \& Gallagher, 2008).

Segundo Field, Diego, Pelaez., Deeds, Delgado, (2012), na Espanha, Inglaterra, Austrália e Estados Unidos, a depressão é a doença mental mais diagnosticada no contexto universitário. Os fatores estressores que permeiam 0 ambiente acadêmico impactam na saúde e qualidade de vida dos estudantes, podendo ser percebidas através da queda de produtividade, dificuldades interpessoais, gerando altos custos pessoais e sociais (Buchanan, 2012). 
Com base nas análises feitas e comparando com os achados de um estudo realizado por Fioravanti, et al. (2006) com estudantes universitários, os quais obtiveram média entre 38,7 e 42,6 na escala IDATE Estado e entre 38,0 e 41,8 na escala IDATE traço observa-se que os níveis de ansiedade estão maiores em comparação ao grupo analisado neste estudo. Sendo os resultados médios encontrados de 43,5 pontos no IDATE Estado e 47,1 no IDATE Traço.

Segundo Ibrahim \& Abdelreheem, (2014), 43,9\% dos estudantes de medicina apresentam sinais significativos de ansiedade e $57,9 \%$ de depressão. Neste mesmo estudo foi observado que $29,3 \%$ dos estudantes de Farmácia apresentam sinais de ansiedade e $51,1 \%$ de depressão. Comparando com - presente estudo encontramos dados parecidos com os estudantes de psicologia sendo que $42 \%$ da amostra com níveis altos na Escala IDATE-Traço e $24 \%$ com níveis moderados ou graves de depressão. Nos participantes deste estudo os níveis de ansiedade chamam a atenção devido à alta porcentagem de estudantes com níveis altos de ansiedade como traço.

Eisenberg et al (2007) sugerem em seu estudo que a prevalência de depressão e ansiedade entre estudantes de graduação foi de $15,6 \%$ e entre estudantes de pós-graduação foi de $13 \%$. Quando comparado aos dados desta amostra nota-se um aumento nestes resultados sendo os sinais de ansiedade traço $42 \%$ (alta) e depressão $9 \%$ (grave).

Segundo informações da World Health Organization (2017), no ano de 2015, a proporção mundial da depressão foi de 4,4\%. Grandes pesquisas feitas com base na população mostram que até $33,7 \%$ desta são afetadas por um transtorno de ansiedade durante a vida (Bandelow, et al 2015). Foi encontrado um percentual de $9 \%$ dos participantes com sintomas depressivos grave.

Os achados deste estudo indicam que os estudantes de Psicologia apresentaram maiores índices de sintomas depressivos grave $(9 \%)$, sendo a população geral $(4,4 \%)$ (Organização Mundial de Saúde 2017). Os sintomas de ansiedade como traço, se apresentaram ainda maiores, índice de $44 \% \mathrm{com}$ nível alto de ansiedade.
Este aumento pode estar relacionado ao ambiente universitário que tem se tornado cada vez mais estressor. Ibrahim, Al- Kharboush, El-Khatib, Al Habib, Asali, (2013) sugerem que as principais causas da ansiedade estão associadas aos cursos acadêmicos condensados e falhas acadêmicas. Os quadros de ansiedade e depressão podem acarretar em abuso de substâncias, ideações suicidas, comportamentos sexuais de risco, dependência de smartphones, além de impactar negativamente no desenvolvimento das habilidades cognitivas e no processo de aprendizagem dos estudantes universitários (Ibrahim, Al- Kharboush, El-Khatib, Al -Habib, Asali, 2013; Mustaffa, Aziz, Mahmood, Shuib, 2014; Boumosleh, Jaalouk, 2017).

O construto ruminação se mostra relacionado com os sintomas de ansiedade $(r=0,692, p<0,01$, correlação forte) e depressão $(r=0,444, p<0,01$, correlação moderada). A presença de pensamentos ruminativos podem predizer a manifestação desses transtornos. O alto nível de ruminação alto pode ser visto como um agravante no processo de adoecimento mental dos estudantes de Psicologia. Alguns estudos apontam que a dificuldade de atualizar as informações e novos conteúdos na mente podem dificultar a regulação emocional (Jonides \& Nee, 2006) aumentando problemas desta ordem. Sánchez, Álvaro, \& Vázquez, Carmelo (2012), sugerem que os indivíduos com depressão, dispõem de formas ineficazes de regulação emocional a situações negativas, favorecendo a manutenção de uma resposta emocional negativa sustentada, favorecendo o desenvolvimento da ruminação e pouca flexibilização comportamental. Pessoas que ruminam muito apresentam dificuldades na resolução de problemas e prolongam seus episódios depressivos (Nolen-Hoeksema, 1991, 2004; Nolen-Hoeksema, Morrow \& Fredrickson, 1993). Os maiores índices de ruminação associados a um alto grau de sintomas de ansiedade podem ter um pior prognóstico na saúde mental dos estudantes, uma vez que a ruminação é uma forma ineficaz de regulação emocional.

Estudos já apontam que quanto maior é o nível de ansiedade e a depressão, maior o será risco em desenvolver problemas sociais, perda de produtividade e diminuição do engajamento em muitas atividades. Clark e Watson (1991) buscam compreender 
a relação entre ansiedade e depressão, devido a presença de sintomatologia inespecífica, uma vez alguns sintomas estão presentes em quadros depressivos e ansiosos, os autores sugerem características comuns relacionadas com a expressão de afetos negativos como tensão e instabilidade.

Ao comparamos os níveis de ansiedade e sintomas de depressão em estudantes do início e fim do curso de psicologia, não foi encontrado diferenças significativas em relação ao período do curso. Nas comparações entre tipo de instituições (pública e privado) ou de gênero (homem e mulher), também não foram encontradas diferenças significativas. Uma hipótese para tentar explicar esse achado é a de que os estudantes de psicologia já apresentam índices mais altos que a população geral de ansiedade e depressão desde o início da vida universitária neste curso. Amaral et al (2008) em um estudo com estudantes de medicina encontrou maior predomínio de depressão entre os alunos do terceiro e do quarto ano. Por outro lado, Velez et al (2008) demonstraram que não existem diferenças significativas entre os níveis de depressão e ansiedade nos alunos no início e no final do curso.

O estudo de Mancevska et al (2008) evidenciou que os alunos de segundo ano apresentaram níveis de depressão menores que os alunos de primeiro ano, no entanto estes apresentavam maiores níveis de ansiedade. A própria literatura demonstra resultados diferentes frente aos níveis de ansiedade e depressão durante o período do curso.

Não foi encontrado diferença significativa ao compararmos a variável gênero assim como nos achados de (Bayram \& Bilgel, 2008; Grant, Marsh, Syniar, Williams, Addlesperger, 2002). Já no estudo feito por Ibrahim \& Abdelreheem, (2014) encontrou uma maior prevalência dos sintomas ansiosos e depressivos em mulheres. No presente estudo, uma limitação importante é o maior número de pessoas do sexo feminino, uma vez que no curso de Psicologia a maioria dos estudantes são do respectivo sexo.

A partir de dados que indicam um aumento dos níveis de depressão e principalmente ansiedade nos estudantes de psicologia é importante pensar em medidas de intervenção. Nos estudos realizados por Wadlinger \& Isaacowitz, (2011) \& Mustaffa,
Aziz, Mahmood, Shuib, (2014) sugerem que algumas medidas não farmacológicas, como o uso de técnicas de flexibilização dos pensamentos, regulação de emoções, relaxamento, foco atencional como Mindfulness e treinamento de habilidades sociais com intuito de prevenir ou amenizar a manifestação de sintomas depressivos e ansiosos podem ser úteis neste processo.

Em resumo o estudo aponta que os níveis de depressão e principalmente ansiedade, nos estudantes de psicologia, estão em um nível maior quando comparados com alguns estudos que investigaram outros cursos universitários. Os níveis de ansiedade e depressão, estão fortemente correlacionados entre si e também com os níveis de pensamentos ruminativos o que indica um agravamento do quadro e maiores dificuldade de regulação emocional dos estudantes. Ao comparamos as diferentes fases (início e fim) do curso de psicologia, não foi encontrado diferenças significativas nos níveis de ansiedade e depressão. Não foi encontrado também diferenças significativas em relação ao gênero ou se a condição da universidade é pública ou particular.

O estudo apresenta algumas limitações. $O$ fato de ser um estudo quantitativo não foi possível entender de maneira mais detalhada os motivos ou relações desses índices encontrados na escala. Por se tratar de uma pesquisa transversal, não é possível acompanhar essas medidas ao longo do tempo ou ao longo do curso de Psicologia. Os participantes do estudo foram escolhidos por conveniência o que pode gerar viés nos resultados encontrados. Outra limitação é a falta de pesquisas que englobam estudantes do curso de Psicologia, para comparações de dados.

\section{Considerações finais}

De acordo com os achados deste estudo, os estudantes de Psicologia apresentam maiores índices de sintomas depressivos e ansiosos que a população geral e estudantes de outros cursos universitários, no entanto estes resultados não se diferenciam quanto ao período do curso, tipo de instituição (publica ou privado) ou gênero. Os dados encontrados nos fazem pensar na hipótese que os estudantes de 
Psicologia já apresentam índices mais altos que a população geral de ansiedade e depressão desde o início da vida universitária neste curso. É difícil definir o fenômeno como sendo inerente ao ambiente universitário ou ligado a especificidades da Psicologia, pois não houve comparação com outros cursos. Seria importante a avaliação desses mesmos índices em outros cursos de graduação para tentar responder a perguntas como está. É interessante realizar estudos futuros buscando essa resposta.

O desenvolvimento de programas de intervenção em grupos objetivando estimular a empatia, pensamentos positivos, trabalhos colaborativos, propondo novas formas de enfrentamento, criação de lembretes com frases agradáveis e reforçadoras, meditação, regulação emocional, incentivo aos exercícios físicos, psicoeducação alimentar, psicoterapia individual e terapias focais de curta duração são exemplos de práticas que reduzem a incidência e intensidade dos sintomas da ansiedade e depressão, uma vez que a saúde física, flexibilização de pensamentos e as boas relações implicam diretamente na saúde mental dos indivíduos.

Os autores sugerem que outros estudos de caráter quantitativo e longitudinal com estudantes de Psicologia sejam realizados, avaliando possíveis causas e acompanhamento destes indivíduos durante a trajetória no curso e comparação de dados.

\section{Contribuição dos autores}

Marília Lopes Oliveira Bezerra: desenho de estudo, discussão dos resultados e redação do artigo. Gustavo Marcelino Siquara: discussão de resultados e redação do artigo. José Neander Silva Abreu: desenho de estudo, discussão de resultados, redação do artigo e supervisão da pesquisa.

\section{Conflitos de interesses}

Nenhum conflito financeiro, legal ou político envolvendo terceiros (governo, empresas e fundações privadas, etc.) foi declarado para nenhum aspecto do trabalho submetido (incluindo mas não limitandose a subvenções e financiamentos, conselho consultivo, desenho de estudo, preparação de manuscrito, análise estatística, etc).

\section{Referências}

Bandelow, B., \& Michaelis, S. (2015). Epidemiology of anxiety disorders in the 21 st century. Dialogues Clin Neurosci, 17(3), 327-335. Recuperado de https://www.ncbi.nlm. nih.gov/pmc/articles/PMC4610617/

Buchanan, J. L. ( 2012). Prevention of depression in the college student population: a review of the literature. Archives of Psychiatric Nursing, 26(1), 21 -42. Recuperado de https://www.ncbi.nlm. nih.gov/pubmed/22284078. doi: 10.1016/i. apnu. 2011.03 .003

Del Porto, J. A. (1999). Conceito e diagnóstico. Revista Brasileira de Psiquiatria, 21 (Supl. 1), 06-11. Recuperado de http://www.scielo.br/pdf/rbp/ v21s1/v21s1a03.pdf. doi: 10.1590/s1516$\underline{44461999000500003}$

Eisenberg D., Gollust S. E., Golberstein E., Hefner J. L. (2007). Prevalence and correlates of depression, anxiety and suicidality among university students. American journal of orthopsychiatry, 77(4), 534-542. Recuperado de https://www.ncbi.nlm.nih.gov/pubmed/18194033. doi: $10.1037 / 0002-9432.77 .4 .534$

Fioravanti, A.C.M., Santos, L.F, Maissonette, S., Cruz, A.P.M., \& Landeira-Fernandez, J. (2006). Avaliação da estrutura fatorial da Escala de Ansiedade-Traço do IDATE. Avaliação Psicológica, 5(2), 217-224. Recuperado de http://pepsic.bvsalud.org/pdf/avp/v5n2/v5n2a 11 . pdf

Gallagher, R. P. (2008). National survey of counseling center directors. The International Association of Counseling Services, Inc. Recuperado de http://d-scholarship. pitt.edu/28169/1/2008_National_Survey_of_ Counseling_Center_Directors.pdf

Guthrie, E. A., Black, D., Shaw, C. M., Hamilton, J., Creed, F. H., Tomenson, B. (1995). Embarking upon a medical career: psychological morbidity in first year medical students. Med Educ., 29(5), 337-41. Recuperado de https://www.ncbi.nlm.nih.gov/pubmed/8699970

Jonides, J., \& Nee, D. E. (2006). Brain mechanisms of proactive interference in working memory. Neuroscience, 139(1), 181-193. Recuperado de https://www.ncbi.nlm.nih.gov/pubmed/16337090. doi: 10.1016/i.neuroscience.2005.06.042 
Aldiabat, K. M., Matani, N. A., \& Le Navence, C-L. (2014). Mental Health among Undergraduate University Students: A Background Paper for Administrators, Educators and Healthcare Providers. Universal Journal of Public Health, 2(8), 209-214. Recuperado de http://www.hrpub.org/download/20141201/UJPH1 17602740.pdf. doi: 10.13189/ujph.2014.020801

Koval, P., Kuppens, P., Allen, N. B., \& Sheeber, L. (2012). Getting stuck in depression: The roles of rumination and emotional inertia. Journal Cognition and Emotion, 26(8): 1412-27. doi: $10.1080 / 02699931.2012 .667392$

MacKean, G. (2011). Saúde mental e bem-estar em ambientes de educação pós-secundária: Uma literatura e uma varredura ambiental para apoiar o planejamento e a ação no Canadá. Associação canadense de serviços universitários e universitários.

Sánchez, Á., \& Vázquez, C. (2012). Sesgos de Atención Selectiva como Factor de Mantenimiento y Vulnerabilidad a la Depresión: Una Revisión Crítica. Terapia psicológica, 30(3), 103-117. Recuperado de https://scielo.conicyt.cl/pdf/terpsicol/v30n3/art10. pdf. doi: $10.4067 /$ S0718-48082012000300010

Wadlinger, H. A., Isaacowitz, D. M. (2011). Fixing our focus: training attention to regulate emotion. Personality and Social Psychology Review, 15(1), 75-102. Recuperado de https://www.ncbi.nlm.nih.gov/pubmed/20435804. doi: $10.1177 / 1088868310365565$

World Health Organization (2017). Depression and other common mental disorders: global health estimates (Site). Recuperado de http://www.who.int/mental_ health/management/depression/prevalence_global_ health_estimates/en/

Zanon, C., Borsa, J. C., Bandeira, D. R., \& Hutz, C. S. (2012). Relações entre pensamento ruminativo e facetas do neuroticismo. Estudos de Psicologia, 29(2), 173 181. Recuperado de http://www.scielo.br/pdf/ estpsi/v29n2/a03v29n2.pdf. doi: 10.1590/S0103$\underline{166 \times 2012000200003}$ 